\title{
Imaging the antiparallel magnetic alignment of adjacent Fe and MnAs thin films
}

\author{
R. Breitwieser ${ }^{1,2}$ M. Marangolo, ${ }^{1, a)}$ J. Lüning, ${ }^{2,3}$ N. Jaouen, ${ }^{3}$ L. Joly, ${ }^{4}$ M. Eddrief, ${ }^{1}$ \\ V. H. Etgens, ${ }^{1}$ and M. Sacchi ${ }^{2,3^{\prime}}$ \\ ${ }^{1}$ INSP, UPMC Paris 06, CNRS UMR 7588, 140 rue de Lourmel, 75015 Paris, France \\ ${ }^{2}$ LCP-MR, UPMC Paris 06, CNRS UMR 7614, 11 Rue P. et M. Curie, 75005 Paris, France \\ ${ }^{3}$ Synchrotron SOLEIL, B.P. 48, 91192 Gif-sur-Yvette, France \\ ${ }^{4}$ Swiss Light Source, Paul Scherrer Institut, 5232 Villigen PSI, Switzerland
}

(Received 14 May 2008; accepted 8 September 2008; published online 25 September 2008)

\begin{abstract}
The magnetic coupling between iron and $\alpha$-MnAs in the epitaxial system Fe/MnAs/GaAs(001) has been studied at the submicron scale, using element-selective $\mathrm{x}$-ray photoemission electron microscopy. At room temperature, MnAs layers display ridges and grooves, alternating $\alpha$ (magnetic) and $\beta$ (nonmagnetic) phases. The self-organized microstructure of MnAs and the stray fields that it generates govern the local alignment between the Fe and $\alpha$-MnAs magnetization directions, which is mostly antiparallel with a marked dependence upon the magnetic domain size. () 2008 American Institute of Physics. [DOI: 10.1063/1.2990755]
\end{abstract}

MnAs films grown on GaAs provide spin-polarized sources for achieving spin injection ${ }^{1}$ and tunnel magnetoresistance in GaAs-based spintronic devices. ${ }^{2}$ Moreover, MnAs films grown on $\mathrm{GaAs}(001)$ display, at room temperature (RT), a self-organized pattern of submicron-wide stripes, ${ }^{3,4}$ alternating the ferromagnetic (FM) hexagonal $\alpha$-MnAs phase with the paramagnetic orthorhombic $\beta$-MnAs phase. (A recent study suggests that $\beta$-MnAs may become antiferromagnetic when stripes form. ${ }^{5}$ ) The stripes are aligned parallel to the $\mathbf{c}$ axis of the $\alpha$-MnAs phase, whose easy magnetization direction is perpendicular to the stripes. The film thickness controls the period of the stripes and the height of the steps between them. ${ }^{3}$ For a given thickness, i.e., for a given period, the sample temperature $T$ determines the relative width of $\alpha$ and $\beta$ stripes. Coupled to a FM material, MnAs can induce complex magnetic behaviors, such as a spin valve effect in self-exchange-biased $\mathrm{MnAs} /(\mathrm{Ga}, \mathrm{Mn}) \mathrm{As}$ FM-metal/FMsemiconductor heterostuctures. ${ }^{6}$

With its regular pattern of submicron wide stripes separated by nanometric steps, $\mathrm{MnAs} / \mathrm{GaAs}(001)$ can be considered also as a self-organized template for the growth of nanometer-thick magnetic layers, whose properties can be controlled by varying $T{ }^{7}$ Within this framework, we prepared ultrathin Fe films on MnAs/GaAs(001) with the purpose of investigating the influence of the stripes on the $\mathrm{Fe}$ magnetic properties. ${ }^{8}$ Making use of the element selectivity and of the magnetic sensitivity of $\mathrm{x}$-ray resonant magnetic scattering (XRMS), we showed that the magnetic alignment between Fe and $\alpha$-MnAs switches from parallel to antiparallel as a function of either $T$ or of the applied field $\left(H_{\text {ext }}\right)$. We interpreted our results by assuming that the effective magnetic field felt by the Fe on top of $\alpha$-MnAs is the sum of $H_{\mathrm{ext}}$ and of a dipolar term $\left(H_{D}\right)$ generated by the underlying FM $\alpha$-MnAs. ${ }^{8}$ Therefore, we expect parallel or antiparallel coupling depending on $H_{\text {ext }}$ being larger or smaller than $H_{D}$, respectively.

In this letter we present an $\mathrm{x}$-ray photoemission electron microscopy (X-PEEM) study of the magnetic coupling be-

${ }^{a)}$ Electronic mail: massimiliano.marangolo@insp.jussieu.fr. tween $\mathrm{Fe}$ and MnAs/GaAs(001). The X-PEEM probes the magnetic coupling at the level of single domains, representing an ideal complement to spatially averaged XRMS analysis. In particular, X-PEEM makes it possible to include the details of the magnetic structure in the analysis via micromagnetic calculations.

A $70 \mathrm{~nm}$ thick MnAs layer was prepared by molecular beam epitaxy on GaAs(001) following the procedure detailed in Ref. 8. The $2 \mathrm{~nm}$ thick Fe layer was deposited on top of the MnAs layer kept at $150{ }^{\circ} \mathrm{C}$. The sample was capped with $1 \mathrm{~nm}$ of $\mathrm{ZnSe}$ plus $2 \mathrm{~nm}$ of Al, for protection against oxidation. The X-PEEM measurements were performed at the SIM beamline of the Swiss Light Source, at RT and with no applied magnetic field.

X-PEEM images are shown in Fig. 1, the energy of the circularly polarized $\mathrm{x}$ rays being tuned to the $\mathrm{Mn}-L_{3}$ (a) and to the $\mathrm{Fe}-L_{3}$ (b) resonance. Both images are in fact the difference between two measurements performed with opposite helicity of the incoming photons, in order to highlight the magnetic structure while minimizing all other sources of contrast. In Fig. 1, the stripes run almost parallel to the vertical direction and photons impinge on the sample at $16^{\circ}$ grazing incidence and normal to the stripes, probing the magnetization along the easy axis of $\alpha$-MnAs. In Fig. 1(a), dark and white areas correspond to $\alpha$-MnAs domains with magnetizations parallel and antiparallel to the photon wave vector, respectively. They are separated by gray thin vertical lines, corresponding to $\beta$ stripes. X-PEEM images show a clear correlation between $\alpha$-MnAs domains in Fig. 1(a) and Fe domains in Fig. 1(b), with inverted light/dark patterns. Fe gray domains correspond mainly to $\beta$ stripes, but also to some of the smallest $\alpha$-MnAs domains. These images confirm, at the level of single domains, the dominant RT antiparallel alignment between $\mathrm{Fe}$ and $\alpha$-MnAs [see also Fig. 2(a)]. For a more quantitative analysis of the images, we define a rate of antiparallel coupling $\Delta$ based on the observation of the $\mathrm{Fe} / \mathrm{MnAs}$ alignment for every $\alpha$-MnAs domain, $\Delta=100 \%$ meaning complete antiparallel alignment. The analysis of the entire images of Fig. 1 yields $\Delta=82 \%$. For a more detailed analysis, we indexed each $\alpha$-MnAs domain of 


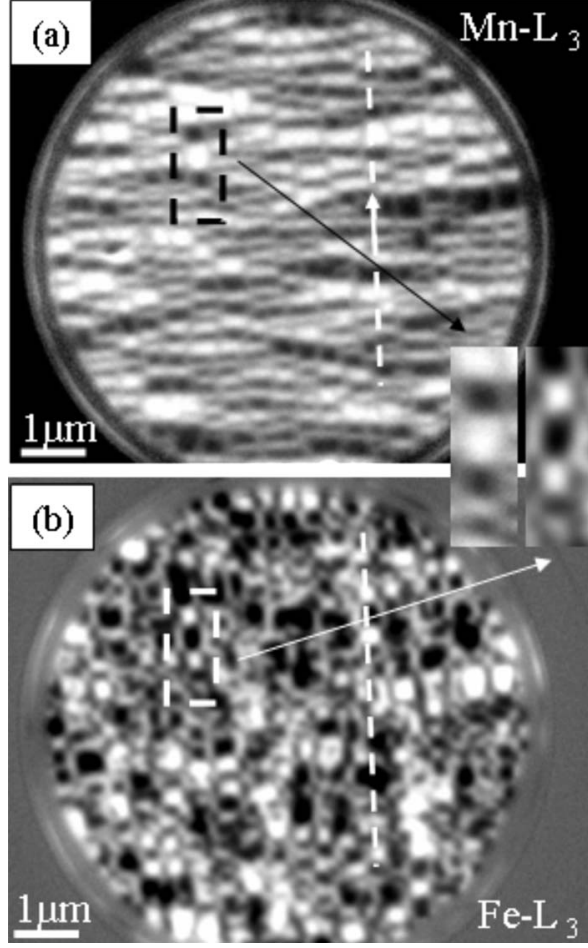

FIG. 1. Magnetic contrast X-PEEM images obtained at the Mn (a) and Fe (b) $L_{3}$ resonance. The areas delimited by rectangles are shown on an enlarged scale. Dashed lines correspond to data shown in Fig. 2(a).

Fig. 1(a) according to its size: the bar diagram of Fig. 2(b) shows $\Delta$ versus the area of the $\alpha$-MnAs domains. While the largest domains $\left(>0.1 \mu \mathrm{m}^{2}\right)$ display a high degree of antiparallel alignment $(\Delta>96 \%)$, this is no longer the case when their size shrinks below $0.06 \mu \mathrm{m}^{2}(\Delta \approx 70 \%)$.

As mentioned before, we interpreted the spatially averaged antiparallel alignment observed by XRMS in terms of the dipolar field generated by $\alpha$-MnAs acting on the $\mathrm{Fe}$ overlayer. ${ }^{8}$ In order to check whether this approach is con-
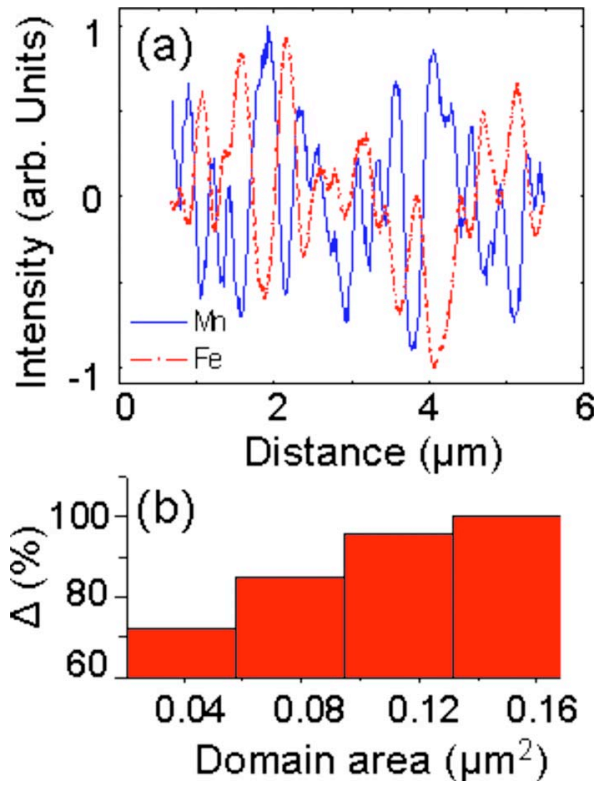

FIG. 2. (Color online) (a) Intensity profiles taken along the dashed lines of Fig. 1, at the $\mathrm{Mn}$ and Fe $2 p$ resonances. (b) $\triangle$, percentage of antiparallel aligned $\mathrm{Fe}$ and $\alpha$-MnAs magnetic domains as a function of the domain area (data from Fig. 1).

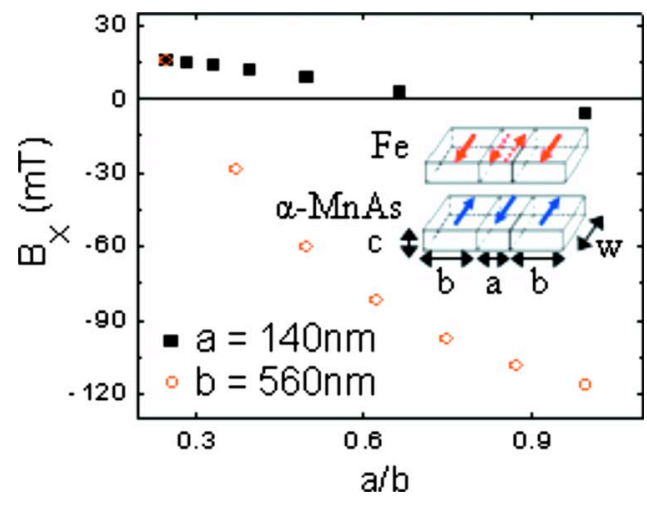

FIG. 3. (Color online) $B_{x}$ is the in-plane component perpendicular to the stripes of the dipolar field generated at the position of the central Fe layer by three $\alpha$-MnAs domains (see sketch drawing). The width of the $\alpha$-MnAs stripe is $\mathbf{w}=280 \mathrm{~nm}$ and its thickness is $\mathbf{c}=70 \mathrm{~nm} . B_{x}$ is calculated as a function of $\mathbf{a} / \mathbf{b}$, either for a constant $\mathbf{a}=140 \mathrm{~nm}$ (squares) or for a constant $\mathbf{b}=560 \mathrm{~nm}$.

sistent with size dependent data of Fig. 2(b), we extended our model to consider a configuration of $\alpha$-MnAs domains as the one depicted in Fig. 3. Three adjacent $\alpha$-MnAs domains pertaining to the same stripe, with alternating magnetization direction, have thickness $c(=70 \mathrm{~nm}$, the layer thickness $)$ and width $w(=280 \mathrm{~nm}$, the average stripe width at RT). Their size along the stripe direction is a for the central domain and b for the two external ones. The orientation of the Fe magnetization on top of the central domain will be influenced by the sum of the dipolar fields generated by all three $\alpha$-MnAs domains, and in particular by its component $B_{x}$ along the easy magnetization direction. The calculations were performed using the Magnetic Bar Calculator on-line code. ${ }^{9}$ Figure 3 shows how the $B_{x}$ component depends on the relative size $\mathbf{a} / \mathbf{b}$ of the central and external domains. By taking a constant $\mathbf{b}=560 \mathrm{~nm}$ and varying $\mathbf{a}$ (open circles), one observes that $B_{x}$ changes sign at around $\mathbf{a} \approx 200 \mathrm{~nm}$. This means that, below this size, the dipolar field generated by the central MnAs domain is overcome by the contributions coming from the neighboring larger domains. Therefore, in the central domain, the overall dipolar field will favor a parallel alignment between Fe and $\alpha$-MnAs. The same can be observed when taking a constant $\mathbf{a}=140 \mathrm{~nm}$ (filled squares) and varying $\mathbf{b}$. The possibility of varying $\mathbf{w}$ by controlling the sample temperature offers an additional parameter that we do not consider here for discussing our RT data.

Finally, Fig. 4 shows magnetic contrast X-PEEM images taken at RT, after annealing the sample in situ at $T=65^{\circ} \mathrm{C}$ (complete transition to the $\beta$-phase). The $\alpha$-MnAs magnetic domains are much larger than in Fig. 1, extending across tens of stripes. Correspondingly, the degree of $\mathrm{Fe} / \mathrm{MnAs}$ antiparallel coupling is very high $(\Delta>96 \%$ over the entire surface $)$, supporting our interpretation outlined above. Similar to Fig. 1(b), one observes that Fe on top of the smallest $\alpha$-MnAs domains can display a gray contrast. The noncollinear alignment of Fe magnetization may happen when the analyzed domain is sandwiched between two nonequivalent size domains and feels a dipolar component normal to the easy magnetization direction.

One important aspect has not been mentioned yet. Our interpretation, as in Ref. 8, requires the hypothesis of a very weak exchange coupling between the two adjacent $\mathrm{Fe}$ and MnAs FM layers. This has been verified experimentally, ${ }^{8}$ but 

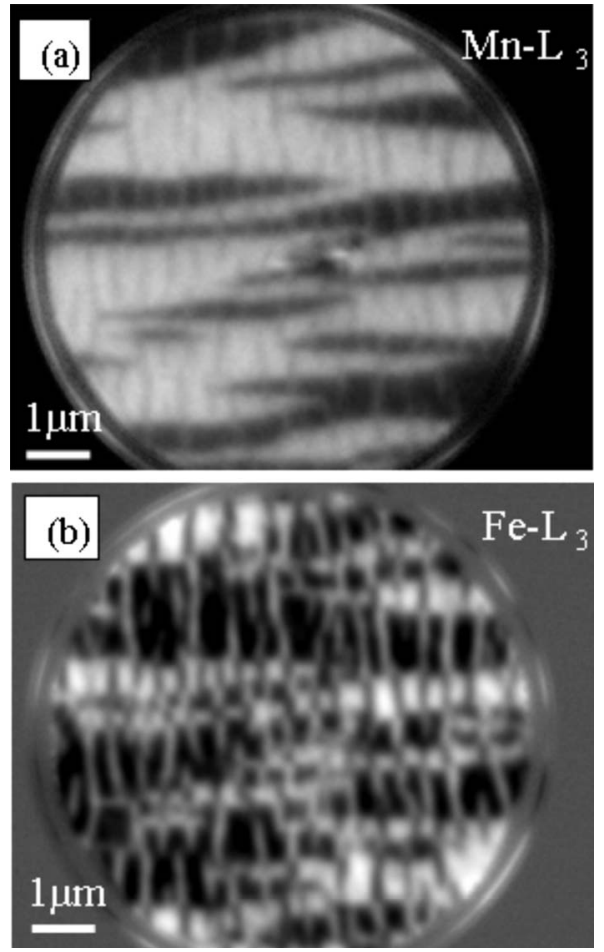

FIG. 4. Same as Fig. 1, after an in situ annealing of the sample at $T=65^{\circ} \mathrm{C}$.

the origin of this weak exchange remains unclear. All our x-ray absorption and dichroism spectra on previous and present samples are characteristic of metallic materials, ${ }^{10}$ with no evidence of oxidation. We cannot exclude, though, the formation of a thin interface layer with antiferromagnetic or ferrimagnetic character, such as $\mathrm{Fe}_{a-x} \mathrm{Mn}_{x} \mathrm{As}{ }^{11}$ or FeMn. ${ }^{12}$

The X-PEEM element-selective magnetic microscopy demonstrates the presence of a dominant antiparallel alignment between $\alpha$-MnAs and Fe at RT, confirming previous spatially averaged XRMS results on $\mathrm{Fe} / \mathrm{MnAs} / \mathrm{GaAs}(001)$. In addition, X-PEEM data show that the degree of antiparallel coupling depends strongly on the size of the magnetic domains, being higher for larger domains. Taking into account the presence of several MnAs domains along a single $\alpha$-stripe, we have interpreted this result within our previous approach based on the influence on the $\mathrm{Fe}$ magnetization direction of MnAs-generated dipolar fields.

We thank F. Nolting and D. Demaille for useful discussions. ANR (project MOMES) and C'Nano-IdF (project TRIPEPS) provided financial support.

${ }^{1}$ J.-M. George, M. Elsen, V. Garcia, H. Jaffrès, and R. Mattana, C. R. Phys. 6, 966 (2005), and references therein.

${ }^{2}$ V. Garcia, H. Jaffrès, J.-M. George, M. Marangolo, M. Eddrief, and V. H. Etgens, Phys. Rev. Lett. 97, 246802 (2006).

${ }^{3}$ L. Däweritz, Rep. Prog. Phys. 69, 2581 (2006), and references therein.

${ }^{4}$ L. Däweritz, C. Herrmann, J. Mohanty, T. Hesjedal, K. H. Ploog, E. Bauer, S. Cherifi, A. Pavlovska, R. Belkhou, S. Heun, and A. Locatelli, J. Vac. Sci. Technol. B 23, 1759 (2005).

${ }^{5}$ E. Bauer, R. Belkhou, S. Cherifi, A. Locatelli, A. Pavlovska, and N. Rougemaille, J. Vac. Sci. Technol. B 25, 1470 (2007).

${ }^{6}$ M. Zhu, M. Wilson, B. Sheu, P. Mitra, P. Schiffer, and N. Samarth, Appl. Phys. Lett. 91, 192503 (2007).

${ }^{7}$ L. N. Coelho, B. R. A. Neves, R. Magalhães-Paniago, F. C. Vicentin, H. Westfahl, R. M. Fernandes, F. Iikawa, L. Däweritz, C. Spezzani, and M. Sacchi, J. Appl. Phys. 100, 083906 (2006)

${ }^{8}$ M. Sacchi, M. Marangolo, C. Spezzani, L. Coelho, R. Breitwieser, J. Milano, and V. H. Etgens, Phys. Rev. B 77, 165317 (2008).

${ }^{9}$ Magnetic Bar Calculator, Integrated Engineering Software, see http:// www.integratedsoft.com/papers/Benchmark/Bar_Magnet.pdf

${ }^{10}$ V. Garcia, Y. Sidis, M. Marangolo, F. Vidal, M. Eddrief, P. Bourges, F. Maccherozzi, F. Ott, G. Panaccione, and V. H. Etgens, Phys. Rev. Lett. 99, 117205, (2007); M. Marangolo, F. Gustavsson, M. Eddrief, Ph. Sainctavit, V. H. Etgens, V. Cros, F. Petroff, J. M. George, P. Bencok, and N. B. Brookes, ibid. 88, 217202 (2002).

${ }^{11}$ T. Kanomata, T. Goto, and H. Ido, J. Phys. Soc. Jpn. 43, 1178 (1977).

${ }^{12}$ F. Offi, W. Kuch, L. I. Chelaru, K. Fukumoto, M. Kotsugi, and J. Kirschner, Phys. Rev. B 67, 094419 (2003). 